\title{
On-line memory polynomial predistortion based on the adapted kalman filtering algorithm
}

\author{
Smail Bachir, Claude Duvanaud and Mourad Djamai \\ University of Poitiers, Laboratoire d'Automatique et d'Informatique Industrielle \\ 42 av. du Recteur Pineau, 86022 Poitiers, France. \\ Emails: sbachir, cduvanaud, mdjamai@iutang.univ-poitiers.fr
}

\begin{abstract}
A new adaptive technique for digital predistortion is presented. The proposed method uses the real-time digital processing of baseband signals to compensate the nonlinearities and memory effects in radio-frequency Power Amplifier. Kalman filtering algorithm with sliding time-window is adapted to track the changes in the PA characteristics. Simulation and measurement results, using digital signal processing, are presented for multicarrier signals to demonstrate the effectiveness of this new approach.
\end{abstract}

\section{INTRODUCTION}

Nonlinear system linearization of microwave components and radio-frequency circuits becomes a challenge and potential useful problem in the radiocommunication system research areas. Interest for Power Amplifier (PA) control is motivated by the increasing growth of the wireless communication systems which has lead to use digital modulation techniques such as (OFDM, BPSK, QPSK, QAM, ...) with non-constant envelope to improve spectral efficiency [1]. As a result of the variable envelope modulation schemes, the improvement of the linearity of the PA becomes an objective of first importance for mobile communication systems [2]. This is due to the nonlinear distortions and dynamical effects which generate unwanted spectrum components for the transmitted signal and lead to Adjacent Channel Power Ratio (ACPR) requirements.

One of the most promising linearization methods for nonlinear PA is to predistort the baseband drive signal [1][3][4][5]. This technique is based on off-line estimation of inverse characteristics of the amplifier to be linearized. If accurate predistortion is required it is necessary to adjust in real-time the predistorter characteristics so that it can track changes in amplifier characteristics such as device aging, temperature changes, supply voltage variations and channel switches [5][6][7]. Kalman Filter (KF) algorithm is one of the most popular adaptive filtering techniques in nonstationary environments and realtime estimation [8][9]. This algorithm, originally developed for linear systems, is generalized for a nonlinear case, called Extended Kalman Filter (EKF). However, the EKF has some inherent limitations mainly due to calculation of complicated analytical derivatives for linearizing the nonlinear model [10]. This is a major constraint for the implementation in adaptive predistortion using nonlinear models with memory. In this article, new approach based on identification by a sliding timewindow is proposed which has less training complexity than standard Kalman Filtering algorithm.
Both analytical and simulation results using memory polynomial predistorter are presented to demonstrate the feasibility and performance of this approach to adaptive predistortion. Also, this paper presents a Preliminary results achieved with an experimental system based on Digital Processing System and a Class $\mathrm{AB}$ amplifier operating close to saturation.

\section{SySTEM MOdeL}

\section{A. Predistorter based on the indirect learning}

The block diagram of indirect learning adaptation is shown in Fig. 1. All signal designations refer either to complex baseband signals, sampled at the period $T_{e}$, and does not depend on the modulation format.

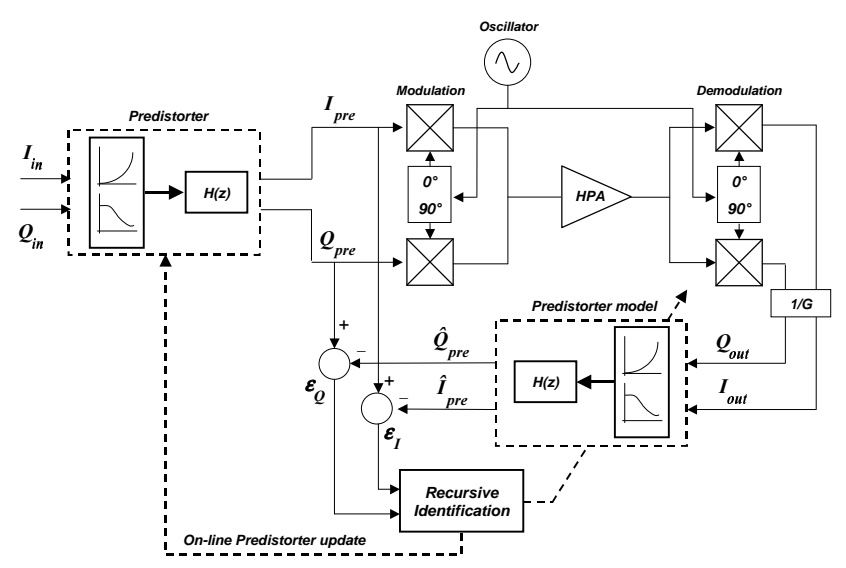

Fig. 1. Baseband equivalent scheme of the adaptive digital predistortion

The predistorter creates a complex predistorted version $V_{\text {pre }_{k}}=I_{\text {pre }_{k}}+j \cdot Q_{\text {pre }_{k}}$ of the transmitted input signal $V_{i n_{k}}=I_{i n_{k}}+j \cdot Q_{i n_{k}}$, based on power amplifier's output $V_{\text {out }_{k}}=I_{\text {out }_{k}}+j \cdot Q_{\text {out }_{k}}$. In the identification part, input and output complex envelopes are sampled for the real-time estimation of the PA inverse function. The input and output signals of the predistorter model are respectively $V_{\text {out }_{k}} / G$ and $\hat{V}_{\text {pre }_{k}}$, where $G$ is the PA gain. The feedback path called "Recursive identification" is the predistorter training based on minimization of the $I Q$ errors $\varepsilon_{I}$ and $\varepsilon_{Q}$. The identification algorithm converges when the multivariable quadratic criterion $J=\varepsilon_{I}^{2}+\varepsilon_{Q}^{2}$ is minimized. After identification, the new predistorter parameters are uploaded in predistorter which becomes an exact copy of predistorter model. 


\section{B. Predistortion model}

Volterra series are used in nonlinear model with memory implemented to a range of applications in system modeling and analysis like channel identification, PA characterization, echo cancellation [1][10][11]. The main advantage of such models is that they are linear-in-parameters allowing Least Mean Square (LMS) estimation techniques. However, there are severe drawbacks, especially for on-line identification, such as the large number and complexity of coefficients depending on the number of kernels (memory and the degree of nonlinearity). A special case of Volterra series is to consider a diagonal representation of their Kernels corresponding to Hammerstein model. This model, used intensively in literature [6][7][10], can be interpreted by a memoryless nonlinearity followed by a filter group delay reducing so the number of coefficients. In this paper, the Hammerstein memory polynomial used for the predistorter block is described as:

$$
V_{p r e_{k}}=\sum_{n=0}^{N-1} \sum_{p=0}^{P} \alpha_{n, 2 p+1} \cdot\left|V_{i n_{k-n}}\right|^{2 p} \cdot V_{i n_{k-n}}
$$

where $P$ is the nonlinearity order, $N$ represents the memory length of the power amplifier and $\alpha_{n, 2 p+1}$ are the predistortion complex coefficients. For parameters estimation, the model (1) is expressed in linear regression system such as:

$$
V_{\text {pre }_{k}}=\underline{\varphi}^{T} \cdot \underline{\theta}
$$

where $\underline{\varphi}^{T}$ is the transposed regression vector of input signal and $\underline{\theta}$ is the vector of parameters to be estimated:

$$
\underline{\varphi}=\left[\begin{array}{c}
V_{i n_{k}} \\
\vdots \\
\left|V_{i n_{k}}\right|^{2 P} \cdot V_{i n_{k}} \\
\vdots \\
V_{i n_{k-N}} \\
\vdots \\
\left|V_{i n_{k-N}}\right|^{2 P} \cdot V_{i n_{k-N}}
\end{array}\right] \text { and } \underline{\theta}=\left[\begin{array}{c}
\alpha_{0,1} \\
\vdots \\
\alpha_{0,2 P+1} \\
\vdots \\
\alpha_{N, 1} \\
\vdots \\
\alpha_{N, 2 P+1}
\end{array}\right]
$$

The objective of identification procedure is to obtain recursively the optimal values of the vector $\underline{\hat{\theta}}$ which minimize $I Q$ errors.

\section{KALMAN FILTERING ALGORITHM WITH SLIDING WINDOW}

The KF algorithm is based on the discrete state space model describing the future evolution of the system when the input is given. In identification approach, the Kalman gain corrects iteratively the estimate according to the error between measured output and desired input [8]. The advantage of this technique is that the estimate is corrected recursively at each iteration. However, there are severe drawbacks, not acceptable in realtime estimation, such as the great number of calculations with complex data and matrix to obtain an appropriate $\mathrm{KF}$ gain [10]. To reduce and simplify these calculations, the proposed method is based on the description of a sliding time-window. In this case, the time domain is decomposed into several data sets as shown in Fig. 2. At the end of each set, the vector of parameters is corrected according to KF algorithm, which amounts to introduce a new sampling period greater than $T_{e}$.



Fig. 2. On-line estimation with sliding window

For this on-line calculation with $N_{w}$ points for each window, we define an $i^{\text {th }}$ quadratic criterion $J_{i}$ based on the $I Q$ errors $\varepsilon_{I}$ and $\varepsilon_{Q}$

$$
J_{i}=\sum_{k=1}^{N_{w}}\left(\varepsilon_{I}^{2}+\varepsilon_{Q}^{2}\right)=\underline{\varepsilon}_{I}^{T} \underline{\varepsilon}_{I}+\underline{\varepsilon}_{Q}^{T} \underline{\varepsilon}_{Q}
$$

By minimization of this criterion, an update unknown vector is calculated from:

$$
\left\{\begin{array}{l}
\underline{\theta}_{i+1}=\underline{\theta}_{i}+P_{i+1} \frac{\phi^{T}}{\sigma_{b}^{2}}\left(\varepsilon_{I}+j \cdot \varepsilon_{Q}\right) \\
P_{i+1}^{-1}=\left(P_{i}+Q\right)^{-1}+\frac{\phi^{T} \phi}{\sigma_{b}^{2}}
\end{array}\right.
$$

where $P$ is the error covariance matrix, $\sigma_{b}^{2}$ is the process noise variance, $Q$ is the parameters covariance matrix and $\phi$ is a regression matrix [12]. Notes that these design parameters will considerably affect the performance of the Kalman algorithm and to get an optimal tracking and fast convergence, these parameters must assume appropriate values [7].

To start the optimization, the initial conditions of the predistorter have to be defined. The initialization is very important because it guaranties stability and high speed convergence. For an unknown amplifier characteristics, we can initialize the vector of Digital Predistortion (DPD) coefficients $\underline{\theta}$ at unity gain values, i.e.:

$$
\underline{\theta}_{0}=\left[\begin{array}{llllll}
1 & 0 & 0 & \cdots & 0 & 0
\end{array}\right]
$$

Another solution is to perform an off-line identification of the predistorter using LMS algorithm [12]. The calculated parameters can be downloaded on the digital signal processor (DSP) and serve as DPD initial values.

It is very important to note that any adaptive scheme for PA linearization is sensitive to the gain value $G$ (Fig. 1). This parameter indicates the power ratio output/input to be applied for signal normalization. Thus, it is necessary to adjust it during estimation to insure algorithm convergence. In our case, this gain is calculated for each transmitted sequence according 
to the maximum input and output power level, and inserted for the next estimation:

$$
G=\sqrt{\frac{P_{o u t}^{\max }}{P_{\text {in }}^{\max }}}
$$

\section{Simulation AND EXPERIMENTAL RESUlts}

In this section, we illustrate through simulations and experiments, performance of the memory polynomial predistorter identified using modified KF algorithm.

\section{A. Simulation results}

The proposed DPD technique is used to linearize an actual Class AB PA (HEMT ZJL-3G), at the frequency of $2.1 \mathrm{GHz}$, modelized by nonlinear model with memory. The test signal is a 16-QPSK digitally modulated signal at rate of $5 \mathrm{Mb} / \mathrm{s}$ and shaped with a raised cosine filter with rolloff factor of 0.25 . We compare the power spectral density (PSD) of input and output signals to evaluate the effectiveness of the predistorter in reducing spectral regrowth. In this part, the predistorter (Eq. $1)$ is defined with two delay taps $(\mathrm{N}=2)$ and $5^{\text {th }}$ odd-order nonlinearity. The power amplifier was driven to the saturation point and then backed off by $1 \mathrm{~dB}$. All results are given with a vector of parameters initialized using LMS algorithm.



Fig. 3. Power spectral density of output signal

Fig. 3 shows the performance improvement in terms of spectral regrowth. The complex predistorter with memory could achieve $20 \mathrm{~dB}$ reduction in spectral distortion.

To simulate a modification of the PA characteristics during transmission, PA parameters are modified at $500 \mu \mathrm{s}$. Figures (4.a) and (4.b) give respectively the evolution of real and imaginary parts of predistorter parameters during linearization procedure. The new DPD values corresponding to the modified amplifier model are achieved in only $300 \mu \mathrm{s}$ corresponding to 15 iterations. Noted that each time-window is composed from $N_{w}=500$ data.
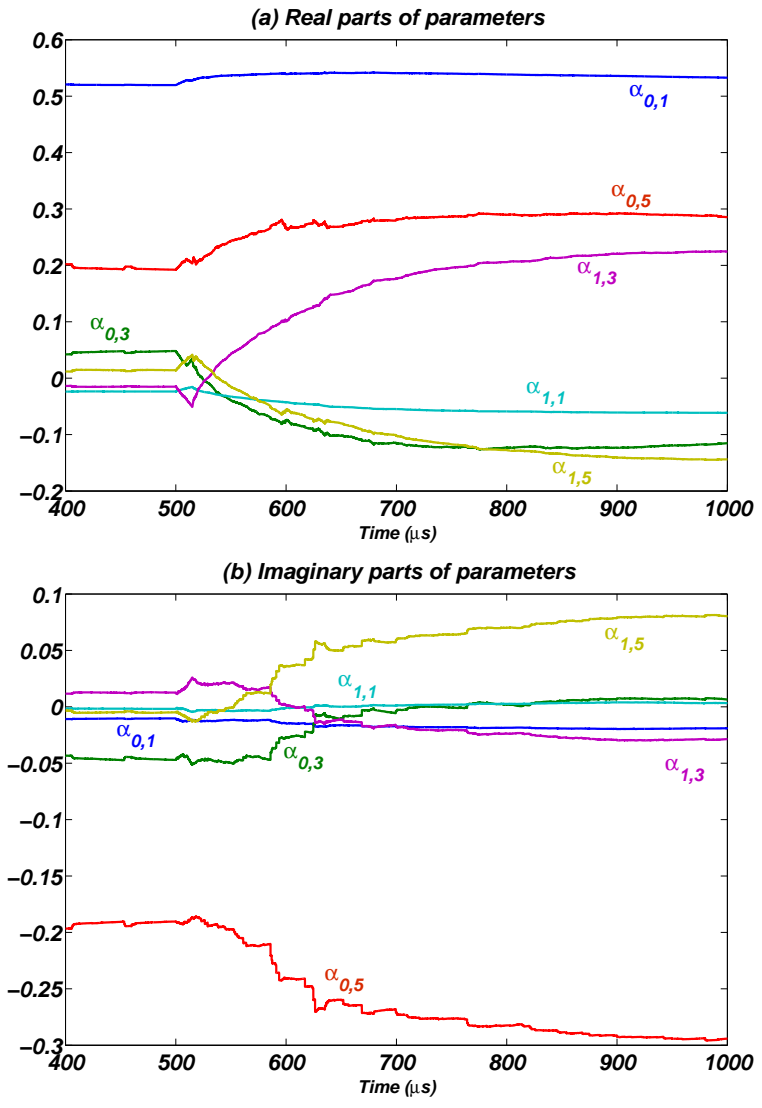

Fig. 4. Evolution of parameters during estimation procedure

\section{B. Experimental results}

This section describes the practical results obtained by the prototype system shown in Fig. 5.

The power amplifier is a commercial Class AB ZHL-42 from MINI CIRCUITS manufacturer. Quadrature modulator AD8349 and demodulator AD8347 are inserted at the input and output of the PA. The DSP processor is a (ADSP21161N) platform with dual DAC/ADC 4 inputs/6 outputs ports. They are standard commercial units from Analog Devices.

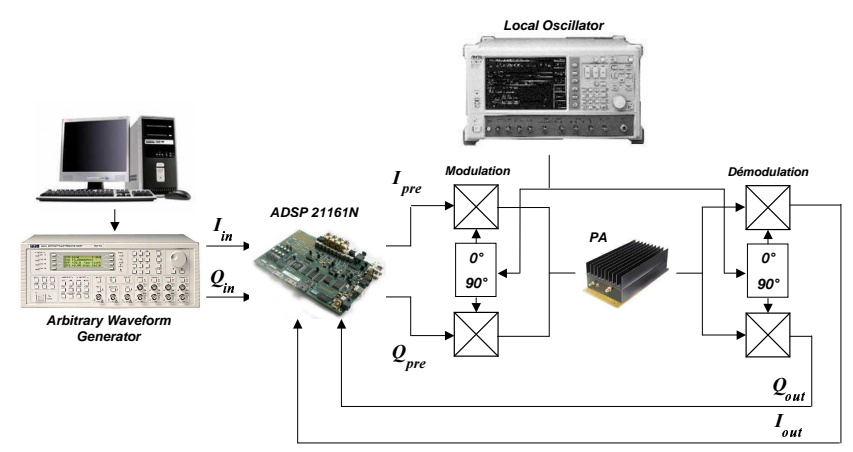

Fig. 5. Experimental setup

Fig. 6 shows the classical two-tone test of the amplifier. In this case, a $1.2 \mathrm{KHz}$ sinusoidal signal is applied at the $I_{\text {in }}$ input 


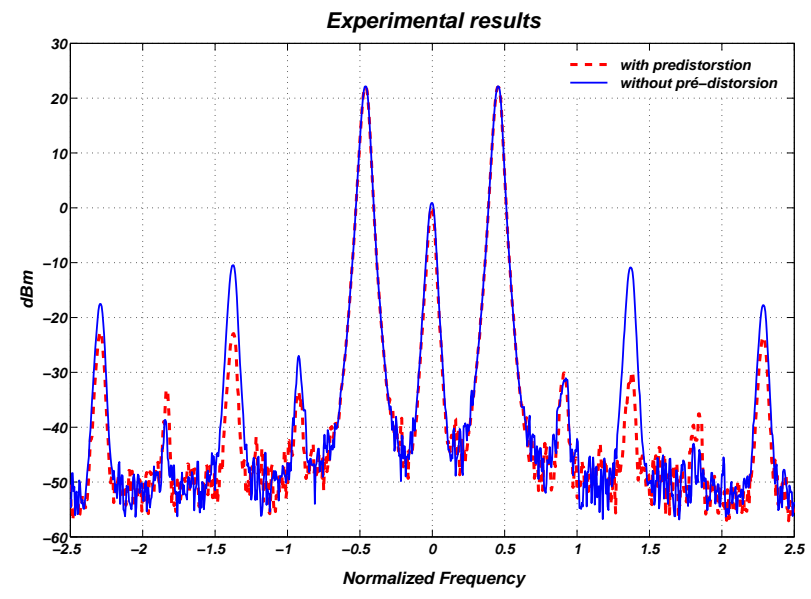

Fig. 6. Power spectral density

and the carrier frequency is fixed at 900MHz. It's important to note that the initial values of the DPD parameters corresponds to a unity gain (5). The real time adjustment of the DPD parameters allows $\sim 15 \mathrm{~dB}$ improvement of the third order intermodulation distortion.

Fig. 7 shows the time domain measurements of predistorted signal and residual $\varepsilon_{I}$. In this figure, we begin the adaptation algorithm at $0.8 \mathrm{~ms}$ and we can observe that after a transient of $2 \mathrm{~ms}$, the estimation error is correctly minimized.
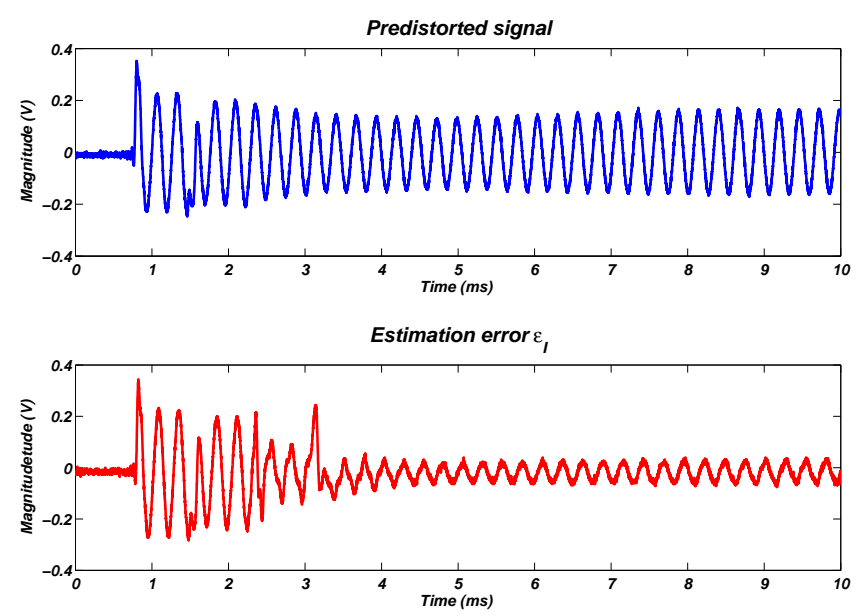

Fig. 7. Time domain measurements of predistorted signal and residual

\section{CONCLUSION}

A new technique for performing baseband predistortion has been described. In this approach, an alternative Kalman Filtering algorithm is introduced to design and estimate a complex predistortion with memory. Identification algorithm has been suitably modified to insure convergence, stability and reduce number of calculation during estimation. The technique uses the real-time transmitted signals through the PA to perform coefficients updates.
The effectiveness of this approach is demonstrated through simulation results, showing that the adapted Kalman Filtering predistorter reduces the adjacent channel interference. The time domain measurement illustrates the capability of this procedure to track PA changes.

A general DPD experimental system based on DSP microprocessor has been build and shows good spectral control, illustrated by an improvement of $\sim 15 \mathrm{~dB}$ of $\mathrm{IM}_{3}$ for a two tone test.

\section{REFERENCES}

[1] P. B. Kenington, High-Linearity RF Amplifier Design, Artech House, London 2000.

[2] A. A. M. Saleh, Frequency-Independent and Frequency-Dependent Nonlinear Models of TWT Amplifiers, IEEE Transactions on Communications, VOL. COM-29, NO. 11, pp. 1715-1720, Nov 1981.

[3] A.A.M. Saleh and J. Salz, Adaptive linearization of power amplifiers in digital radio systems, Bell System Tech. J. VOL. 62, NO. 4, pp. 10191033, April 1983.

[4] Y. Nagata, Linear amplification technique for digital mobile communications, $39^{\text {th }}$ Proc. IEEE Vehicular Tech. Conf. pp. 159-164, San Francisco 1989.

[5] J. K. Cavers, Amplifier linearization using a digital predistorter with fast adaptation and low memory requirements, IEEE Trans. Vehicular Technology, VOL. 39, NO. 04, pp. 374-382, Nov. 1990.

[6] R. Marsalek, P. Jardin, G. Baudoin, From post-distortion to pre-distortion for power amplifiers linearization, IEEE Communications Letters, VOL. 07, pp. 308-310, July 2003.

[7] T. Elgeryd, Iterative algorithms for linearising non-linear systems by digital predistortion, Master thesis report, Royal Institute of Technology, Stockholm 2002.

[8] R. E. Kalman, A new approach to linear filtering and prediction problems, ASME Journal of Basic Engineering, VOL. 82, NO. D, pp.34-45, 1960.

[9] A. E. Nordsjö, An algorithm for adaptive predistortion of certain timevarying nonlinear high-power amplifiers, RADAR 2002, pp. 469-473, 15-17 Oct 2002.

[10] A. V. Malipatil, Y.-F. Huang, Amplifier predistortion using unscented Kalman filtering, IEEE $6^{t h}$ Workshop on Signal Processing Advances in Wireless Communications, pp. 221-225, Juin 2005.

[11] H. Ku, M.D. McKinley and J.S. Kenney, Quantifying memory effects in $R F$ power amplifiers, IEEE Transactions on Microwave Theory and Techniques, VOL. 50, NO. 12, pp. 2843 - 2849, December 2002.

[12] L. Ljung, System identification: Theory for the user, Prentice Hall, USA, 1987. 